\title{
Research on the convergence architecture of 5G and industrial communication
}

\author{
Zhen Wang ${ }^{1, *}$, Dantao Han $^{1}$, Yanjie Gong ${ }^{1}$, and Yanling Zhao ${ }^{1}$ \\ ${ }^{1}$ Instrumentation Technology and Economy Institute (ITEI), Beijing 100055, China
}

\begin{abstract}
As the next generation of mobile communication technology, $5 \mathrm{G}$ has the characteristics of enhanced mobile broadband (eMBB), massive machine-type communication (mMTC), and ultra-reliable low latency communication (URLLC), which are critical technologies for the future development of industrie 4.0. So far, these technologies have been explored and applied in industry, however, there is still a lack of general architectures. This paper presents a $5 \mathrm{G}$ and industrial communication convergence architecture and elaborates on the implementation methods from the aspects of device access technology and network configuration strategies, and discusses the transparent transmission and mapping methods adopted by $5 \mathrm{G}$ terminal access technology as well.
\end{abstract}

\section{Introduction}

The emergence of smart factories requires industrial wireless communication technology to have better performance in terms of bandwidth, delay, reliability, etc. As a new generation of wireless communication technology, 5G took the high demands of industrial applications into consideration at the beginning of its design. eMBB can meet the transmission needs of industrial big data, such as visual inspection, predictive maintenance, etc. URLLC can facilitate highly critical applications with very demanding requirements in terms of end-to-end (E2E) latency, reliability and availability. However, integrating 5G into the existing industrial system and combining $5 \mathrm{G}$ with the current industrial communication technology remains a problem for industrialists.

After the release 15 of $5 \mathrm{G}$ technology in 2018, studies on application of $5 \mathrm{G}$ in industry began to emerge. Researchers analyzed the remarkable impact of 5G technology in advancing industrial change from the perspective of industrial evolution and proposed typical industrial application scenarios of 5G technology [1]. The integration and application of 5G technology and existing industrial communication technology will be the main direction of the future 5G industrial applications according to [2] and [3], in which the implementation of $5 \mathrm{G}$ and industrial network hybrid topology architecture is proposed. In [4], the 5G system and TSN technology are further analyzed and the improvements of 5G network are proposed to meet industrial end-to-end application requirements. But until now, the research of $5 \mathrm{G}$ and industrial communication integration application is still at an early stage. $5 \mathrm{G}$ technology is mainly used as the link of industrial communication to realize the

* Corresponding author: wangzhen@,tc124.com 
transparent transmission of industrial data, which is relatively simple. Based on the analysis of wired and wireless communication technology, this paper proposes a $5 \mathrm{G}$ and industrial communication technology integration application architecture and several application scenarios. The research is still in its infancy, but it is expected to contribute to the industrial application of $5 \mathrm{G}$ technology.

This paper is divided into four sections. The rest is organized as followed, section 2 deals with the theoretical foundations for the development of the research, including realtime communication technology, 5G network architecture and protocol. Section 3 describes $5 \mathrm{G}$ and industrial communication technology integration application architecture and use cases. In the end, the conclusion along with the next steps are presented in section 4 .

\section{Approach}

With the involvement of automation, process control and artificial intelligence, industrial systems have required more sophisticated real-time and reliable communication infrastructure. For now, the current industrial network technology can easily meet these requirements, such as CC-LINK, PROFINET RT/IRT. Industrial wireless networks have been widely investigated to support industrial application, for example, zigbee, WI-FI, Bluetooth, etc. However, the high interference in industries makes the desirable communications reliability very challenging. After the release 15 of $5 \mathrm{G}$, the proposed feature in $5 \mathrm{G}$ networks offers a potential technology to industrial systems with the assurance of low-latency and reliable communications, but integrating $5 \mathrm{G}$ with industrial communication technology is still a problem. This chapter introduces the current technical solutions of industrial communication and focuses on the analysis of the $5 \mathrm{G}$ network architecture and communication protocol.

\subsection{Industrial ethernet networks}

In the last decade, some of the well-known and commonly implemented wired communications solutions can offer the desired real-time, reliability and availability, such as Ethernet/IP, PROFINET RT, EtherCAT and CC-Link IE, etc. In order to fulfill real-time and reliability, the industrial Ethernet network technologies adopted various approaches to the IP and/or MAC layer, achieving the deterministic communication. Industrial Ethernet network technologies can be divided into three classes to meet different requirements of indutries network performance. The first class uses standard Ethernet hardware and standard TCP/IP software stacks for industrial communication. As the simplest approach, it is widely used in industry field, e.g. Ethernet/IP and Modbus TCP. The second class uses standard Ethernet controllers, in stead of using TCP/IP stacks, it deploys a professional data protocol for communication, such as PROFINET RT, POWERLINK and EtherCAT. The third class uses dedicated hardware down to MAC layer, such as EtherCAT, PROFINET IRT, Sercos III and CC-Link IE [5].

As can be seen from above, Industrial communication technology is based on the TCP/IP protocol. However, the TCP/IP protocol stack is designed mainly for the reliability of communication, and a large number of redundant mechanisms are used, hence the realtime performance can not meet the needs of industrial applications. As the TCP/IP protocol conforms with OSI model, the application data need to pass through the transport layer, IP layer and MAC layer step by step, so as to realize the real-time and reliability demands of industrial application, such as PROFINET RT and IRT. The 5G technology should reference the evolution of industrial wired network technology to meet more demanding industrial application requirements. 


\subsection{G system architecture}

The 5G system architecture described in [6] was explained, see Figure 1. The data of the user equipment is first sent to the $\mathrm{gNB}$, then the $5 \mathrm{G}$ core network, and finally the Internet. Between the user equipment and the gNB, the data is transmitted through wireless signals. Between the gNB and the core network, the data is transmitted through optical fiber networks.

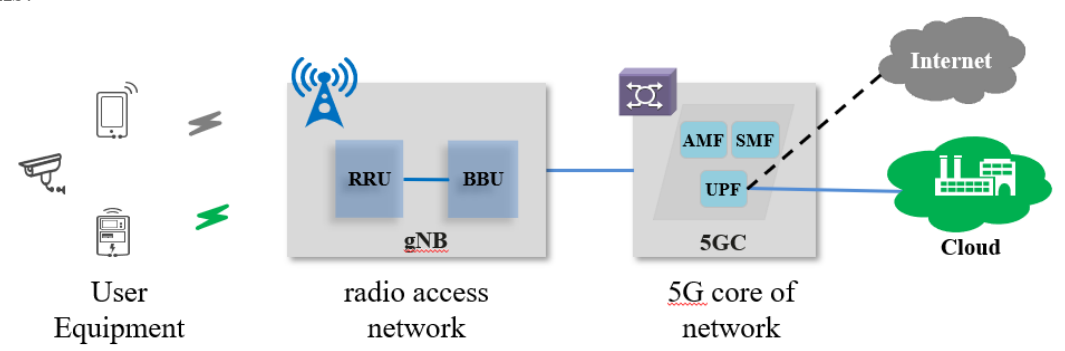

Fig. 1. The typical scenario of the $5 \mathrm{G}$ system.

The abstract representation of the $5 \mathrm{G}$ system is shown in Figure 2. It mainly explains the $5 \mathrm{G}$ system architecture from the perspective of $5 \mathrm{G}$ functions. the Access and Mobility Management Function (AMF) is in charge of registration and connection management functions. The Session Management Function (SMF) is in charge of establishment, modification and release of PDU session including tunnel management between the (R)AN node and the User Plane Function (UPF). The UPF is in charge of downlink and uplink packet buffering and data notification triggering as well as packet routing and forwarding [7].

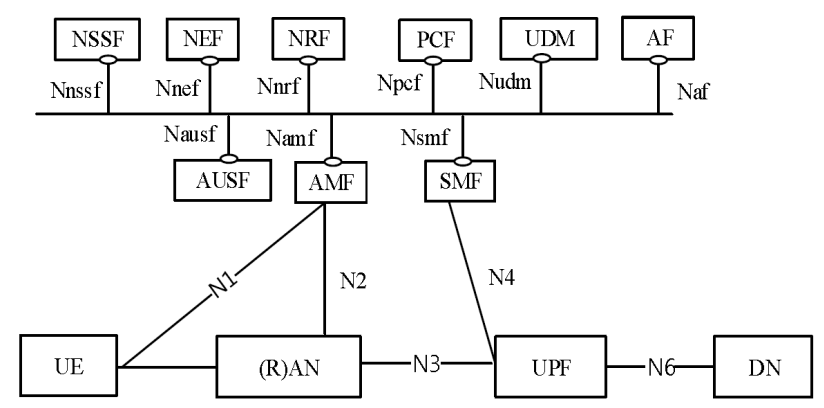

Fig. 2. The function model of $5 \mathrm{G}$ System.

As can be seen from figure 2, the original intention of $5 \mathrm{G}$ network is to realize separation of the control plane and the data plane, minimum dependencies between the Access Network (AN) and the Core Network (CN), flexible and efficient network slicing in addition to decouple of the "compute" resource and the "storage" resource, based on which $5 \mathrm{G}$ systems establish the next generation mobile communication network. Thereby it can support eMBB, mMTC, and URLLC.

\section{$2.35 \mathrm{G}$ communication protocol}

5G communication protocol is separated to the control plane and the data plane, as shown in Figure 3 and 4 respectively. The user equipment side of $5 \mathrm{G}$ communication protocol mainly consists of PHY, MAC, RLC and PDCP, on the higher level, SDAP operations will be performed on the data plane, while RRC operations will be performed on the control 
plane. In addition, the $5 \mathrm{G}$ protocol architecture and corresponding functions are explained as below.

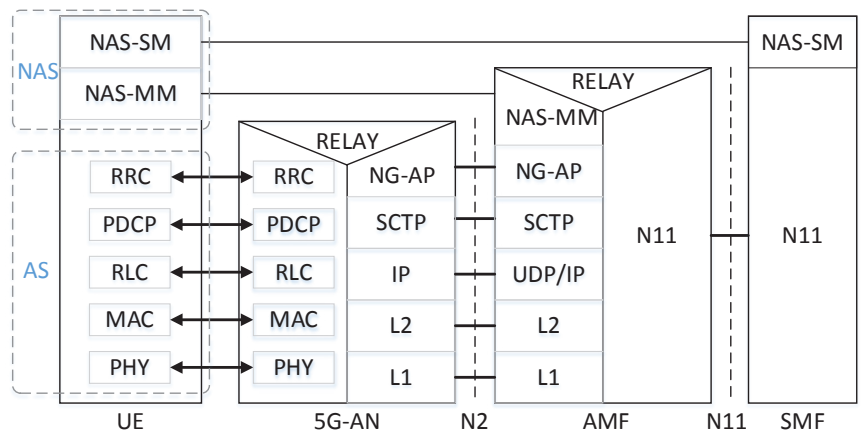

Fig. 3. Control plane protocol.

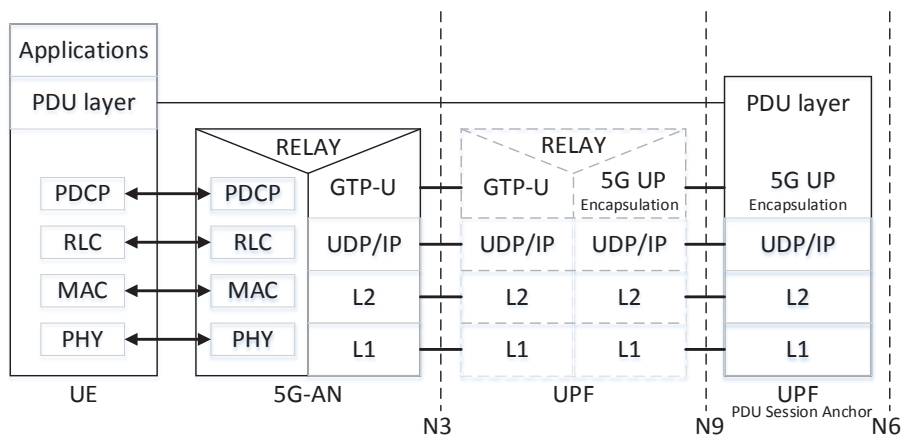

Fig. 4. Data plane protocol.

As Figure 5 shows, RRC is in the control plane while SDAP is in the user plane. PDCP is responsible for IP header compression and decompression as well as encryption and decryption of the data over the radio interface. Next, PDCP feeds the stack down to the RLC layer, which is responsible for error correction with ARQ, concatenation and segmentation, in sequence delivery and protocol error handing. Down to the MAC layer, which is responsible for multiplexing and de-multiplexing, hybrid ARQ error correction, scheduling and transport format selection. Finally, the physical layer is responsible for modulation and demodulation of physical channels, frequency and time synchronization, power weighting of physical channels, MIMO antenna processing and RF processing [8].

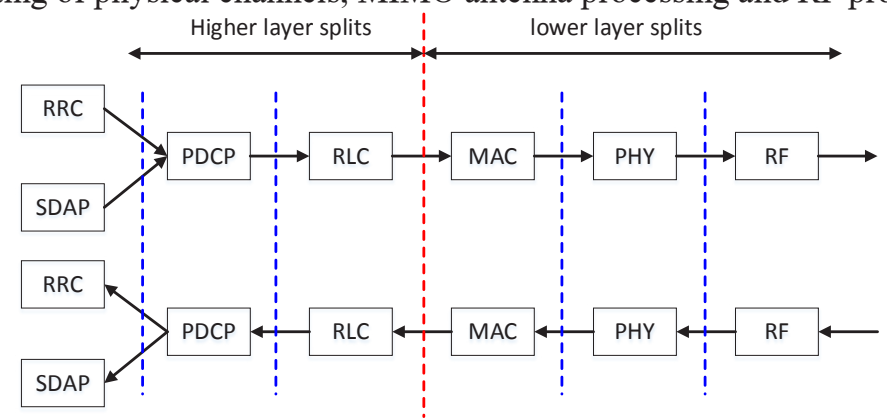

Fig. 5. 5G protocol architecture. 
By analyzing the $5 \mathrm{G}$ network architecture and communication protocol and comparing $5 \mathrm{G}$ wireless network with the wired industrial communication network, the $5 \mathrm{G}$ system architecture can be simplified as shown in Figure 6 . The $5 \mathrm{G}$ system can be a router and the control function of the $5 \mathrm{G}$ network can be routing rules. Meanwhile, $5 \mathrm{G}$ communication protocol can be decomposed according to the OSI standard. SDAP and RRC are communication application layers, PDCP and RLC are IP layers, and the MAC layer and PHY layer have the same functions. Based on this theory, a new architecture for the integration of $5 \mathrm{G}$ and industrial communication applications is proposed.

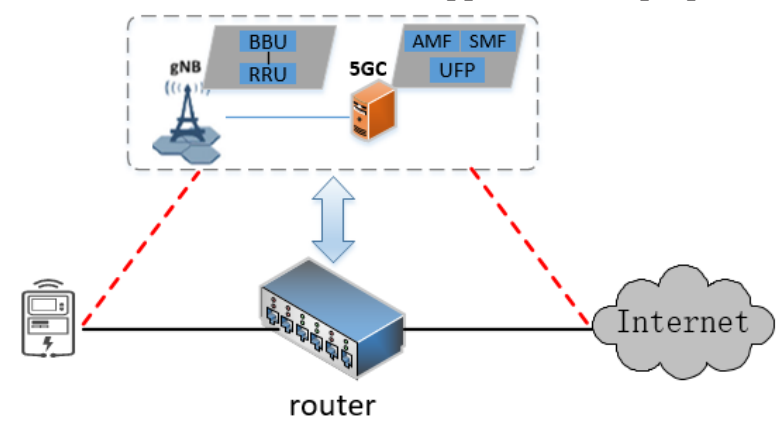

Fig. 6. Abstract model of $5 \mathrm{G}$ system architecture.

\section{Results}

\subsection{Converged architecture}

The integrated architecture of $5 \mathrm{G}$ technology and industrial communication includes two aspects. In terms of $5 \mathrm{G}$ network architecture, the control plane of the $5 \mathrm{G}$ system can provide high-precision synchronous clocks and functional adaptation modes according to the business needs of industrial applications, while the data plane of the $5 \mathrm{G}$ system can realize fusion transmission with industrial communication data, priority transmission and real-time transmission and provide different types of data carrying capabilities as well, see Figure 7.

In terms of communication protocol, some flexible use fields are provided at the protocol layer to realize flexible configuration of network functions. Thus, it is compatible with different industrial communication technologies and can realize functions such as priority transmission of data, real-time transmission and clock synchronization of user terminal equipment.

In practice, the converged architecture is mainly embodied in $5 \mathrm{G}$ network system adaptation technology and user terminal convergence technology. In Figure 8, the user terminal as the basis for the integration of industrial communication, needs to be compatible with different industrial communication technologies. When the user terminal initiates different service requests, the $5 \mathrm{G}$ network system performs functional reconfiguration to meet different industrial applications demands. 


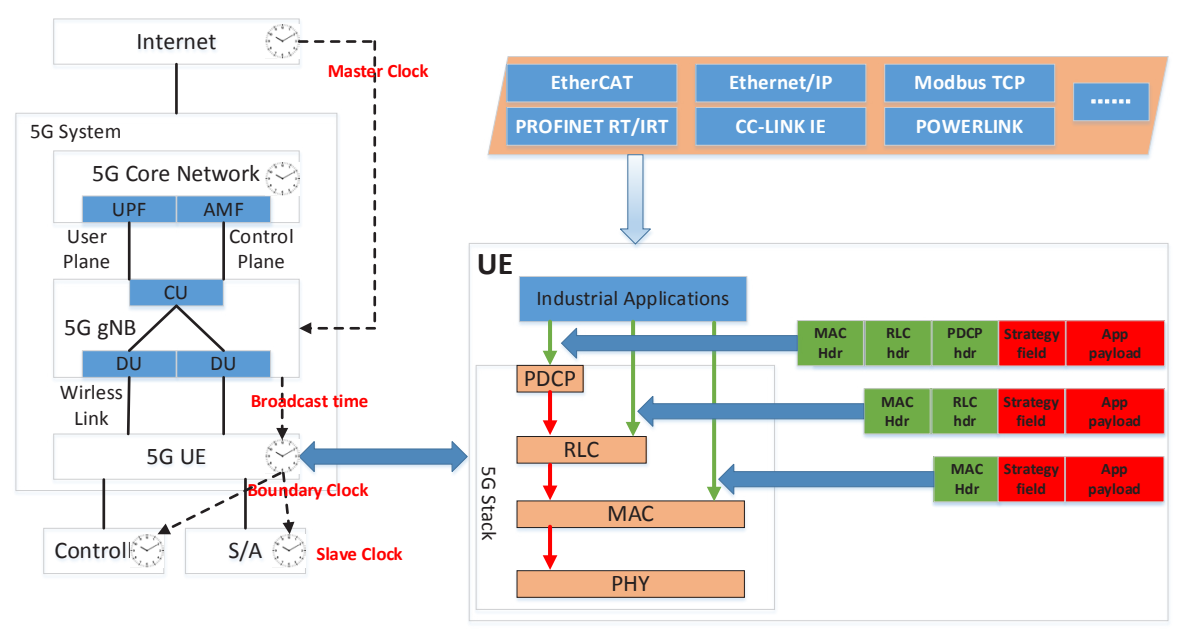

Fig. 7. Converged architecture of $5 \mathrm{G}$ system.

\subsection{Terminal access}

For the $5 \mathrm{G}$ user terminal integration application mentioned above, two technologies can be used to achieve the deep integration of $5 \mathrm{G}$ technology and industrial communication, namely, transparent transmission and mapping of industrial communication.

\subsubsection{Transparent transmission}

5G transparent transmission technology, that is, $5 \mathrm{G}$ as a channel for industrial data transmission, which can realize transparent transmission of industrial data from the industrial application layer, IP layer and MAC layer on the $5 \mathrm{G}$ network. For example, if industrial communication adopts the standard TCP/IP protocol, the source IP layer data packet can roam on the $5 \mathrm{G}$ system and be delivered to the destination through transparent transmission. After that, the source IP layer data packet is reconstituted at the destination to complete the TCP/IP protocol analysis and application layer processing, see Figure 8 . The transparent transmission method can realize the seamless integration of $5 \mathrm{G}$ and the existing industrial communication network system and the rapid upgrade and transformation of the industrial system. At present, transparent transmission is widely adopted for the integration of $5 \mathrm{G}$ and industrial applications. 


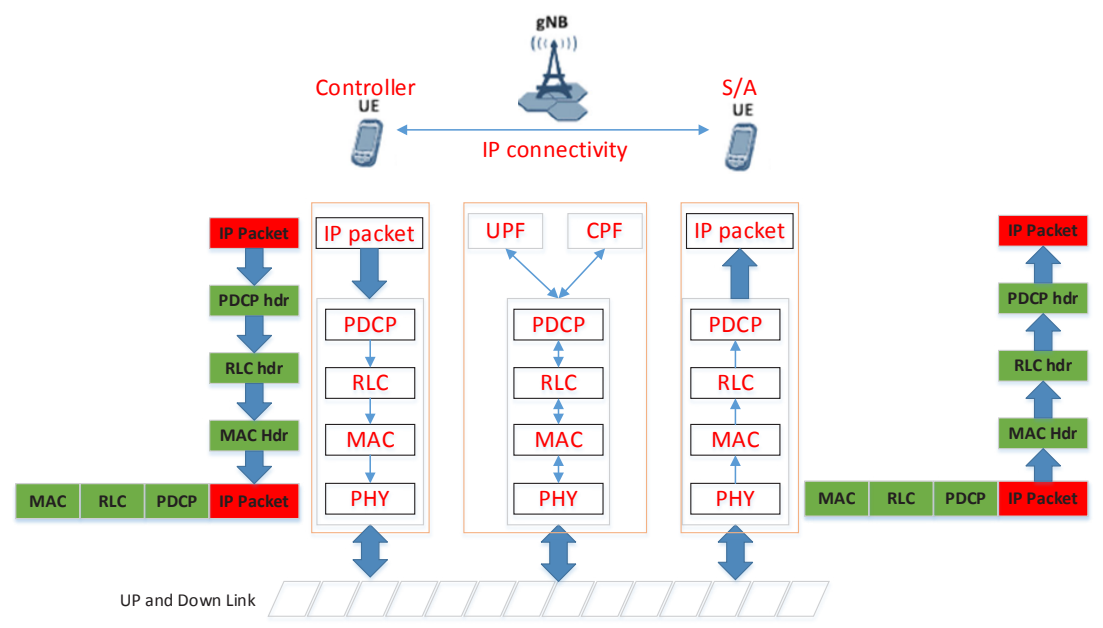

Fig. 8. Data flow diagram of transparent transmission (from controller to s/a).

\subsubsection{Mapping}

The mapping technology of $5 \mathrm{G}$ aims to realize the requirements of industrial communication technology on the $5 \mathrm{G}$ system, such as data priority scheduling mechanism, time scheduling strategy, etc., thereby $5 \mathrm{G}$ technology can meet the priority transmission and delay of industrial applications. Compared with transparent transmission technology, $5 \mathrm{G}$ mapping combines the requirements of industrial communication technology with the mechanism of $5 \mathrm{G}$, which can give full play to the technical advantages of $5 \mathrm{G}$. For example, the $5 \mathrm{G}$ network is used as the MAC layer of the industrial communication network, see Figure 9. The priority scheduling and delay strategy of the industrial network communication technology are mapped to the transmission mechanism of the $5 \mathrm{G}$ network, hence the priority of industrial applications is guaranteed through the $5 \mathrm{G}$ network transmission, which can greatly shorten the processing of real-time messages in the protocol stack and improve real-time performance. So far, some organizations have carried out the mapping research work of $5 \mathrm{G}+\mathrm{OPC} \mathrm{UA}, 5 \mathrm{G}+\mathrm{TSN}$ and other related technologies. 


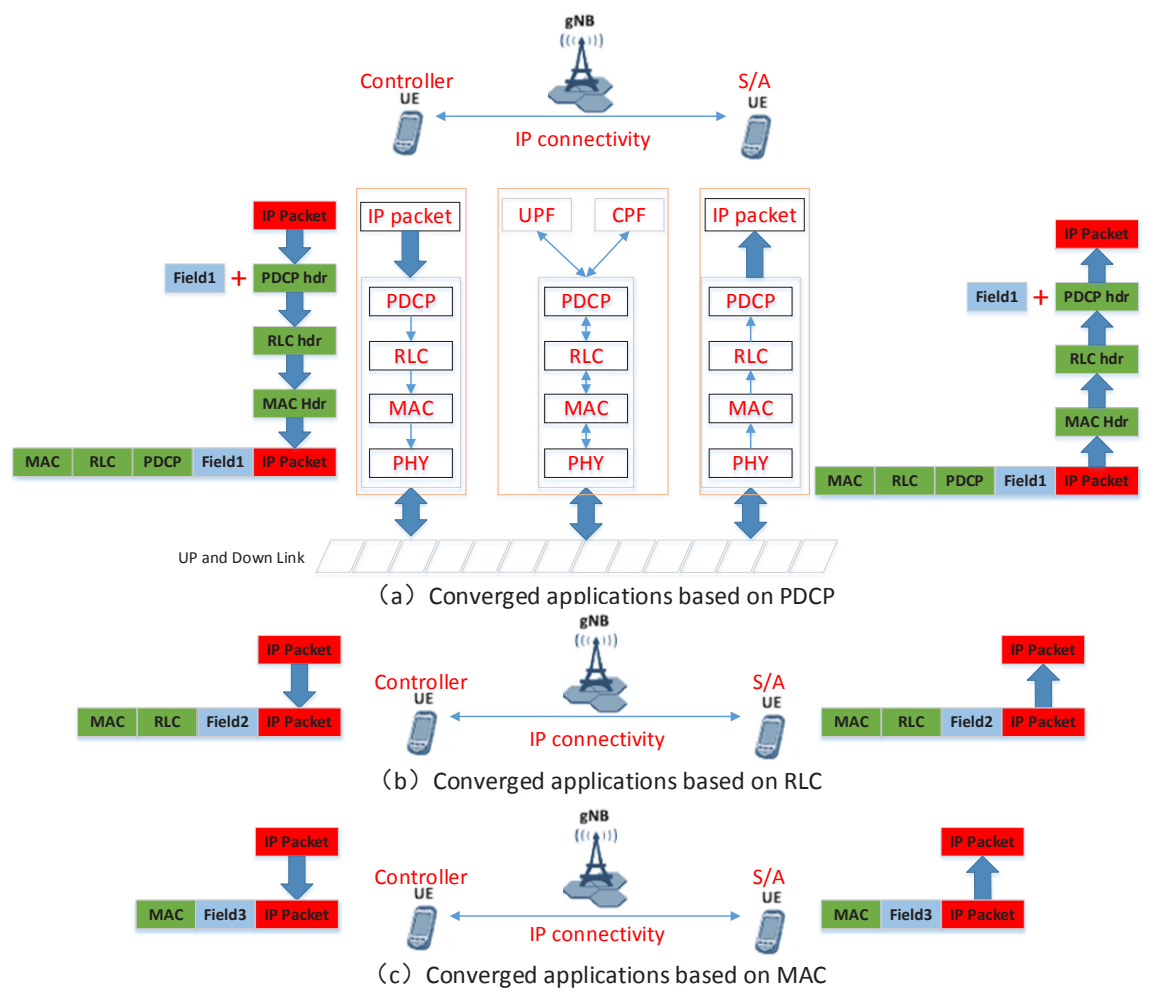

Fig. 9. Data flow diagram of mapping (from controller to $s / a$ ).

\subsection{Control rules}

With 5G fusion application technology, user terminal can realize the rapid access of industrial communication, but it cannot completely solve the problems of 5G industrial application, such as synchronous clock, priority etc. 5G network should also support various business needs of the industry, just like industrial routers, using excellent routing strategies and clock synchronization mechanisms to support industrial applications, which can be introduced to $5 \mathrm{G}$ networks, including:

- Synchronous clock strategy, realizes the time synchronization of the equipment in the $5 \mathrm{G}$ system, which is a key indicator for realizing deterministic communication in industrial applications. There are two solutions for clock synchronization. One is that the $5 \mathrm{G}$ system periodically initiate a system time synchronization calibration function to calibrate the terminal equipment and routing equipment connected to the $5 \mathrm{G}$ system, the other is the user terminal initiate clock synchronization when connecting to the $5 \mathrm{G}$ network for the first time.

- Routing strategy, the $5 \mathrm{G}$ system use different communication routing strategy identifiers for different types of communication services as a basis to mobilize the resource allocation of the $5 \mathrm{G}$ system for industrial applications. For transparent transmission services, system resources can be easily configured. For high-real-time services, network slicing resources and edge computing resources are allocated, and the UPF function is sunk to the device side, etc. By recombining different resources, $5 \mathrm{G}$ system can meet real-time requirements of industrial applications. 
To realize the industrial application of $5 \mathrm{G}$ technology, there are still many difficulties. Industrial applications could make full use of $5 \mathrm{G}$ network and its communication protocol to achieve the integration of industrial communication and 5G. At the same time, use terminal access mode and $5 \mathrm{G}$ configuration strategy to flexibly organize the $5 \mathrm{G}$ network to solve practical problems.

\section{Conclusion}

Some of the existing industrial Ethernet technologies have been able to provide solutions that meet the high real-time and reliability requirements of industrial applications. Learning from its success and combining with the characteristics of $5 \mathrm{G}$ technology, this paper proposed a converged architecture of $5 \mathrm{G}$ and industrial communication and introduced the implementation methods from the aspects of device access technology and network configuration strategies, meanwhile, the transparent transmission and mapping methods adopted by $5 \mathrm{G}$ terminal access technology was introduced. The transparent transmission technology can reuse the existing industrial communication technology and equipment, while making minor changes to $5 \mathrm{G}$ and making full use of the existing $5 \mathrm{G}$ technologies such as network slicing, QoS etc., thus satisfying the needs of industrial applications such as flexible networking and flexible production. Therefore, transparent transmission technology of $5 \mathrm{G}$ is the main direction of the integration of $5 \mathrm{G}$ and industrial communication in a short time. The mapping methods of 5G technology, as long as equipped with a dedicated $5 \mathrm{G}$ communication module for network frame analysis and processing, it can meet the strict real-time requirements of industrial applications. Currently, transmission and mapping methods of 5G technology have been explored and applied in some industrial fields, however the control strategy for industrial applications of 5G systems still needs to be improved, which can be a subject for future research.

This research was supported by a grant from the National key research and development Project "Research on Real-time Analysis and Processing Technology of Instrument and Meter Edge Computing Data" (Grant No. 2018YFB2003503), and Chongqing technology Innovation and Application development Project "Development and application of industrial iot equipment for intelligent factories" (Grant No. cstc2019jscx-fxydX0026).

\section{References}

1. Rao, Sriganesh, K., Prasad, \& Ramjee. (2018). Impact of 5g technologies on industry 4.0. Wireless Personal Communications An Internaional Journal.

2. Neumann, A., Wisniewski, L. , Ganesan, S. S., Rost, P. , \& Jasperneite, J. . (2018). Towards integration of Industrial Ethernet with 5G mobile networks. (pp.1-4).

3. Raza, M. , Hussain, S. , Aslam, N. , Hoa Le cm inh, \& Nguyen, H. X. . (0). Industrial Wireless Sensor Networks and 5G Connected Industries. Enabling 5G Communication Systems to Support Vertical Industries. John Wiley \& Sons, Ltd.

4. Khoshnevisan, M. , Joseph, V. , Gupta, P. , Meshkati, F. , Prakash, R. , \& Tinnakornsrisuphap, P. . (2019). 5g industrial networks with comp for urllc and time sensitive network architecture. IEEE Journal on Selected Areas in Communications, 37(4), 947-959.

5. 3GPP SA WG2, "3GPP TS 23.501: System Architecture for the 5G System"

6. Lee, J. , Moon, S. J. , Bae, B. , \& Lee, J. . (2018). Local Area Data Network for 5G System Architecture. (pp.141-146). 
7. Sutton, A. . (2019). Enabling the Verticals of 5G: Network Architecture, Design and Service Optimization. Enabling 5G Communication Systems to Support Vertical Industries.

8. Choi, Y. I. , \& Park, N. . (2017). Slice architecture for 5G core network. Ninth International Conference on Ubiquitous \& Future Networks. IEEE.

9. Wisniewski, L., Schumacher, M., Jasperneite, J. , \& Schriegel, S. . (2012). Fast and simple scheduling algorithm for PROFINET IRT networks. 2012 9th IEEE International Workshop on Factory Communication Systems (WFCS). IEEE.

10. Dopatka, F. , \& Wismuller, R. . (2007). Design of a Realtime Industrial-Ethernet Network Including Hot-Pluggable Asynchronous Devices. Industrial Electronics, 2007. ISIE 2007. IEEE International Symposium on. IEEE.

11. Boubakri, W. , Abdallah, W. , \& Boudriga, N. . (2018). An Optical Wireless Communication Based 5G Architecture to Enable Smart City Applications. 2018 20th International Conference on Transparent Optical Networks (ICTON).

12. Rao, R. M. , Fontaine, M. , \& Veisllari, R. . (2018). A Reconfigurable Architecture for Packet Based 5G Transport Networks. 2018 IEEE 5G World Forum (5GWF). IEEE.

13. Gupta, A. , \& Jha, R. K. . (2015). A survey of 5g network: architecture and emerging technologies. IEEE Access, 3, 1206-1232.

14. Festag, A. . (2015). Standards for vehicular communication-from ieee $802.11 \mathrm{p}$ to $5 \mathrm{~g}$. E \& I Elektrotechnik Und Informationstechnik, 132(7), 409-416.

15. Costa-Requena, J. , Estevan, C. B. , \& Borenius, S. . (2019). Transport layer and Synchronization for Smart Grid and Industrial Internet in $5 \mathrm{G}$ Networks. IEEE International Conference on Communications, Control, and Computing Technologies for Smart Grids. IEEE.

16. Thomas, \& Zemen. (2018). Wireless 5g ultra reliable low latency communications. E \& I Elektrotechnik Und Informationstechnik. 